\title{
Detection of Interstitial Lung Disease in Systemic Sclerosis through Partitioning of Lung Transfer for Carbon Monoxide
}

\author{
Julien Pernot ${ }^{a} \quad$ Eve Puzenat $^{b} \quad$ Nadine Magy-Bertrand ${ }^{c} \quad$ Philippe Manzoni $^{d}$ \\ Anne Gondouin $^{\mathrm{e}}$ Hubert Bourdin ${ }^{\mathrm{a}}$ Marie-Laure Simon-Rigaud ${ }^{\mathrm{a}}$ \\ Jacques Regnarda, ${ }^{a}$ Bruno Degano ${ }^{a, f}$
}

Departments of a Physiology, ${ }^{b}$ Dermatology, ${ }^{c}$ Internal Medicine, ${ }^{\mathrm{d}}$ Radiology and ${ }^{\mathrm{e}}$ Respiratory Diseases, University Hospital, and ${ }^{\mathrm{f}} \mathrm{EA}$ 3920, Faculty of Medicine, University of Franche-Comté, Besançon, France

\section{Key Words}

Systemic sclerosis $\cdot$ Interstitial lung disease $\cdot$ Pulmonary diffusion capacity Pulmonary membrane conductance • Pulmonary capillary blood volume

\begin{abstract}
Background: Interstitial lung disease (ILD) is a leading cause of death in systemic sclerosis (SSc). Sensitivities and specificities of the current pulmonary function tests (PFTs) for the detection of ILD in SSc are poor. Objective: To determine whether diffusion capacity of the lungs for carbon monoxide (DLCO) partitioned into membrane conductance for CO (DmCO) and alveolar capillary blood volume (Vcap) could provide more sensitive clues to ILD than current PFTs. Methods: DmCO and Vcap were determined in 35 consecutive SSC patients in whom a cardiac and/or pulmonary vascular abnormality had been rejected according to the recommended screening algorithm. ILD was diagnosed with high-resolution computed tomography. Results: Among 35 patients [6 men; median age (first-third quartile) 61.9 years (49.567.7)], 22 had no ILD and 13 did. Total lung capacity (TLC), vital capacity and DLCO [percentage of predicted value (\%pred)] were lower in patients with ILD [86 (82-103) vs. 106
\end{abstract}

(98-112), $\mathrm{p}=0.01,96$ (88-112) vs. 114 (104-121), $\mathrm{p}=0.04$, and $67(59-81)$ vs. 80 (71-94), $\mathrm{p}=0.02$, respectively]. DmCO (\%pred) and the ratio of DmCO to Vcap were much lower in patients with ILD [54 (48-72) vs. 83 (66-92), $\mathrm{p}<0.001$, and $0.22(0.21-0.27)$ vs. $0.40(0.35-0.53), \mathrm{p}<0.0001$, respectively]. According to receiver operating characteristic analysis, the DmCO:Vcap ratio displayed higher sensitivity and specificity than TLC, vital capacity and DLCO in identifying ILD in our study group $(p<0.01)$. Conclusions: These results suggest that the partitioning of DLCO might be of interest for identifying ILD in SSc patients.

Copyright $\odot 2012$ S. Karger AG, Basel

\section{Introduction}

Systemic sclerosis (SSc) is a fibrosing autoimmune disease in which interstitial lung disease (ILD) acts as one of the leading causes of death $[1,2]$. The prevalence of ILD associated with SSc (SSc-ILD) may vary from 16 to $100 \%$, depending on the clinical subtype of SSc and the methods selected to define ILD [3]. SSc-ILD is often asymptomatic, and clinical manifestations occur at a late stage of the disease. Early diagnosis and prompt therapy might be

\section{KARGER}

Fax +41613061234

E-Mail karger@karger.ch

www.karger.com (c) 2012 S. Karger AG, Basel

0025-7931/12/0846-0461\$38.00/0

Accessible online at:

www.karger.com/res
Bruno Degano, MD, PhD

Physiologie-Explorations Fonctionnelles

CHU Jean Minjoz

FR-25030 Besançon Cedex (France)

Tel. +33 38121 1807, E-Mail bruno.degano@univ-fcomte.fr 
beneficial from a prognostic standpoint, and systematic screening for SSc-ILD is thus recommended [3]. Recognition of SSc-ILD depends on the diagnostic methods used, increasing from approximately $30 \%$ with chest radiography to approximately $90 \%$ with high-resolution computed tomography (HRCT) [4]. HRCT is the most sensitive and specific tool for the detection of SSc-ILD and is reliable, noninvasive and reproducible $[5,6]$. However, HRCT is not repeated systematically for screening purposes because of cost and exposure to ionizing radiation. Pulmonary function tests (PFTs) are used for screening purposes, as alterations in PFTs often precede symptoms or changes in chest radiography [7]. However, normal spirometry cannot rule out early ILD, as deteriorated PFTs are found in only approximately $50 \%$ of patients with early SSc-ILD [8]. Moreover, as noted by others, PFTs are limited by the wide range of normal values ( $80-120 \%)$; thus, in an individual patient, a result equal to $80 \%$ of the predicted value may not have changed from premorbid levels but may, equally, represent a fall of up to $40 \%$ [9].

Even in asymptomatic SSc patients, PFTs [including spirometry and single-breath diffusion capacity of the lungs for carbon monoxide (DLCO)] and a Doppler echocardiography are recommended at least annually in addition to clinical examination [10]. A standardized algorithm based on symptoms and Doppler echocardiography allows early diagnosis of pulmonary arterial hypertension (PAH) and left ventricular (LV) abnormalities, which are two major complications of SSc $[10,11]$. PFTs are regarded as limited by the wide range of normal values (80-120\%), calculated from the age, gender and height of the patient [9]. Among PFTs, a reduction in DLCO is one of the earliest detectable functional abnormalities in SScILD [3]. However, reduced DLCO is also a hallmark of $\mathrm{PAH}$ and LV failure $[12,13]$. Moreover, the specificity of a decrease in DLCO is poor, as SSc patients without PAH and/or an LV abnormality and/or ILD may have a DLCO as low as approximately $65 \%$ of the predicted value [14].

DLCO can be partitioned into two transfer components or conductances, namely membrane conductance for CO (DmCO), which reflects the diffusion properties of the alveolar capillary membrane, and CO loading on hemoglobin $(\mathrm{Hb})$, which is the product of the $\mathrm{CO}-\mathrm{Hb}$ chemical reaction rate $(\theta \mathrm{CO})$ and the mass of $\mathrm{Hb}$ in the alveolar capillary blood volume (Vcap). Because these two conductances are in series, they are related by the equation proposed by Roughton and Forster [15]: $1 / \mathrm{DLCO}=1 / \mathrm{DmCO}+1 / \theta$ Vcap. A decreased DmCO is interpreted as a 'thickening' of the alveolo-capillary membrane or as a decrease in lung area, and a decreased
Vcap is viewed as a lowered blood volume in the ventilated alveoli [16].

It has been established that the currently recommended screening algorithm enables the diagnosis of PAH and/or LV failure with appropriate sensitivity and specificity $[10,11]$. The current study was designed to examine whether partitioning DLCO might be more accurate than DLCO alone to detect SSc-ILD in those SSc patients without any identified cardiac and/or pulmonary vascular abnormality.

\section{Methods}

\section{Patients}

The study was conducted in consecutive patients referred either to the Department of Dermatology or to the Department of Internal Medicine of the University Hospital of Besançon for evaluation of SSc between June 2010 and May 2011. The disease was classified as of the diffuse cutaneous or limited cutaneous subtype, according to the current classification system [17]. All patients underwent a physical examination, laboratory testing (including a complete blood cell count and antinuclear, anticentromere and anti-SCL-70 antibody assessments), HRCT of the chest, PFTs including spirometry and DLCO and Doppler echocardiography. Systolic left heart failure was defined by an LV ejection fraction $<55 \%$ and diastolic heart failure as an early diastolic mitral annular velocity $<10 \mathrm{~cm} / \mathrm{s}$ associated with a ratio of mitral peak velocity of early filling to early diastolic mitral annular velocity $>15$. Right heart catheterization (RHC) was performed in patients with a tricuspid regurgitation velocity jet of more than $3 \mathrm{~m} / \mathrm{s}$ and in patients with unexplained dyspnea along with a tricuspid regurgitation velocity jet of more than $2.5 \mathrm{~m} / \mathrm{s}$ [10]. The following patients were excluded from the study: patients with pulmonary hypertension defined by a mean pulmonary artery pressure higher than $25 \mathrm{~mm} \mathrm{Hg}$ as measured during RHC and patients with LV failure defined by a pulmonary artery occlusion pressure higher than $15 \mathrm{~mm} \mathrm{Hg}$.

Healthy subjects with no history of cardiopulmonary disease and with normal chest radiography and physical examination findings served as control subjects.

The study protocol was approved by the Ethics Committee of our institution (CPP Est II). All subjects gave their informed consent.

\section{Pulmonary Function Tests}

Forced expiratory volume in $1 \mathrm{~s}$, forced vital capacity, vital capacity and total lung capacity (TLC) were assessed with standard PFT equipment (Sensor Medics, Yorba Linda, Calif., USA). Measurements were performed according to the European Respiratory Society guidelines.

DLCO was measured by a single-breath method breathing room air of $21 \% \mathrm{O}_{2}$ and inhalation of a gas mixture $[0.3 \% \mathrm{CO}$, $0.3 \%$ methane $\left(\mathrm{CH}_{4}\right)$ and $21 \%$ oxygen $\left(\mathrm{O}_{2}\right)$ balanced with nitrogen] from residual volume to TLC followed by a 10 -second breath hold, as recommended. The two components of DLCO, $\mathrm{DmCO}$ and Vcap, were determined using the following equation: $1 / \mathrm{DLCO}=1 / \mathrm{DmCO}+1 / \theta \mathrm{Vcap}$. The reaction rate of $\mathrm{CO}$ with $\mathrm{Hb}$ 
Table 1. Characteristics of patients and control subjects

\begin{tabular}{lccc}
\hline & $\begin{array}{l}\text { SSc-ILD- } \\
(\mathrm{n}=22)\end{array}$ & $\begin{array}{l}\text { SSc-ILD+ } \\
(\mathrm{n}=13)\end{array}$ & $\begin{array}{l}\text { Controls } \\
(\mathrm{n}=16)\end{array}$ \\
\hline Age at PFTs, years & $56.4(48.0-64.5)$ & $66.3(55.2-73.2)^{*}$ & $52.2(39.8-59.5)$ \\
Height, cm & $163(156-170)$ & $159(157-165)$ & $24.9(20.3-29.1)$ \\
Body mass index & $24.6(21.8-28.6)$ & $23.7(21.1-28.3)$ & $11 / 2$ \\
Female/male & $18 / 4$ & $4 / 9^{*}$ & - \\
Limited SSc/diffuse SSc & $19 / 3$ & 2 & $-12 / 4$ \\
Ever smoked & 3 & $10.0(2.8-19.4)$ & - \\
Duration of SSc, years & $9.1(3.0-18.4)$ & 9 & 0 \\
Presence of lung crackles & 1 & 7 & - \\
Presence of abnormalities on chest radiography ${ }^{1}$ & 0 & $2 / 7 / 4^{*}$ & - \\
Anticentromere antibodies/anti-SCL-70 antibodies/others & $13 / 3 / 6$ & $13.1(12.7-13.6)$ & - \\
Hemoglobin, g/dl & $13.8(13.4-14.0)$ & & - \\
\hline
\end{tabular}

Results are expressed as medians (first-third quartile) for continuous variables and otherwise as numbers.

${ }^{*} \mathrm{p}<0.05$ versus SSc-ILD-.

${ }^{1}$ Chest radiography was considered abnormal in the presence of interstitial opacifications and/or areas of attenuation. No other abnormalities were identified.

$\theta$ is proportional to the $\mathrm{Hb}$ concentration. $1 / \theta$ is assumed to be a linear function of alveolar oxygen tension $\left(\mathrm{P}_{\mathrm{A}} \mathrm{O}_{2}\right.$; in $\left.\mathrm{kPa}\right)$, as follows: $1 / \theta=[\mathrm{Hb}$ ref $] /[\mathrm{Hb}] \times\left[\left(0.001 \times \mathrm{P}_{\mathrm{A}} \mathrm{O}_{2}\right)+1.034\right]$, where $[\mathrm{Hb}$ ref] is $14.6 \mathrm{~g} / \mathrm{dl}$ in men and $13.4 \mathrm{~g} / \mathrm{dl}$ in women and $[\mathrm{Hb}]$ is the subject's $\mathrm{Hb}$ concentration in $\mathrm{g} / \mathrm{dl}$. DLCO was also measured under hyperoxic conditions with a gas mixture containing $0.3 \% \mathrm{CO}$, $0.3 \% \mathrm{CH}_{4}$ and $80 \% \mathrm{O}_{2}$. A plot of $1 / \mathrm{DLCO}$ against $1 / \theta$ was obtained, and $1 / \mathrm{DmCO}$ was given by the $\mathrm{y}$-intercept and $1 /$ Vcap by the slope of the straight line. The linearity of the relation had been previously verified in healthy controls using three different oxygen concentrations (21, 50 and $80 \%)$. All measurements were obtained in duplicate. Results were expressed as absolute values and as percentages of predicted values calculated using the reference equations described by Zanen et al. [18].

\section{Chest HRCT Scans}

HRCT scans were obtained using a standardized protocol. Briefly, patients were scanned with 1- or 2-mm collimation at 10 $\mathrm{mm}$ intervals from lung apices to bases during suspended end inspiration while in the prone position without intravenous contrast. Images were reconstructed with a high spatial frequency algorithm. The following HRCT scan findings were recorded: pure ground-glass opacities (defined as increased lung attenuation in the absence of reticular interstitial thickening or architectural distortion); lung fibrosis (defined as reticular intralobular interstitial thickening, traction bronchiectasis, bronchiectasis or any combination thereof), and honeycomb cysts (defined as clustered air-filled lung cysts with contiguous walls) [19]. HRCT scans were read independently by two experimental radiologists blinded to the results of PFTs. HRCT was performed within the 6 months before or after PFTs. Patients without any HRCT scan finding related to ILD were assigned to the SSc-ILD- group, and patients with at least one finding related to ILD were assigned to the SSc-ILD+ group.

Detection of Interstitial Lung Disease in Systemic Sclerosis

\section{Statistical Analyses}

The number of patients was decided according to the principal objective of the study, which was to estimate the predictive capacities and in particular the sensitivity of the ratio of DmCO to Vcap for the detection of ILD among patients with SSc. This number has been linked to the precision of the expected sensitivity [14, $20,21]$. It has been found that a sample of 30 patients (with SSc and without any cardiac involvement) is necessary in order to obtain a precision of $\pm 15 \%$ of the sensitivity (binomial law, confidence interval at 95\%) in the diagnosis of ILD [22].

Data are expressed as medians (1st-3rd quartile) for continuous variables and numbers (percentages) for categorical variables. Comparisons between more than two groups were performed with the Kruskal-Wallis test. Comparisons between two groups were performed with the Mann-Whitney $U$ test. A $\chi^{2}$ test was used for differences in frequency.

Receiver operating characteristic (ROC) curves were constructed to illustrate the power of different PFT indexes to detect ILD over an entire range of cutoff values. The overall diagnostic accuracies of the tests were estimated using the area under the ROC curve.

A p value $<0.05$ was considered significant. Analysis was performed using Statview, version 5.0 (SAS Institute, Cary, N.C., USA).

\section{Results}

\section{Patients}

Forty-nine patients with SSc were screened between April 2010 and March 2011. Among them, 3 had LV failure, 3 had PAH, 1 had PAH and ILD, 4 refused to participate and 3 were unable to perform valid PFTs (1 with 
Table 2. Comparison of PFTs between SSc-ILD+ patients, SSc-ILD- patients and control subjects

\begin{tabular}{lccc}
\hline & SSc-ILD- $(\mathrm{n}=22)$ & SSc-ILD+ $(\mathrm{n}=13)$ & Controls $(\mathrm{n}=16)$ \\
\hline TLC, \%pred & $106(98-112)$ & $86(82-103)^{*}$ & $110(99-116)$ \\
VC, \%pred & $114(104-121)$ & $96(88-112)^{*}$ & $114(107-117)$ \\
FEV $/$ VC, \% & $76(73-79)$ & $75(73-80)$ & $79(76-83)$ \\
DLCO, \%pred & $80(71-94)$ & $67(59-81)^{*}$ & $95(86-104)$ \\
KCO, $\mathrm{ml} / \mathrm{min} / \mathrm{mm} \mathrm{Hg/l}$ & $4.03(3.65-4.79)$ & $19.15(3.41-4.45)$ & $4.75(4.42-5.03)$ \\
DmCO, $\mathrm{ml} / \mathrm{min} / \mathrm{mm} \mathrm{Hg}$ & $30.4(27.4-39.8)$ & $67(59-81)^{*}$ & $43.7(37.9-48.2)$ \\
DmCO, \%pred & $80(71-94)$ & $82.0(77.0-96.3)$ & $102(91-112)$ \\
Vcap, $\mathrm{ml}$ & $70.5(59.0-87.0)$ & $129(118-142)^{*}$ & $85.5(70.3-91.0)$ \\
Vcap, \%pred & $103(95-125)$ & $0.22(0.21-0.27)^{*}$ & $117(96-135)$ \\
DmCO:Vcap ratio & $0.40(0.35-0.53)$ & & $0.52(0.49-0.60)$ \\
\hline
\end{tabular}

\footnotetext{
Values are expressed as medians (first-third quartile).

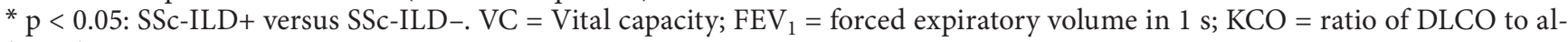
veolar volume.
}

ILD and 2 without any cardiac or pulmonary complication). The 35 remaining patients were studied. Among them, 22 had a normal HRCT scan (SSc-ILD- group), whereas 13 had HRCT features of ILD (SSc-ILD+ group). The abnormal HRCT findings in this group included lung fibrosis in 10 patients, areas of pure ground-glass opacities in 11 patients and/or honeycomb cysts in 5 patients.

Comparison of characteristics between SSc-ILD- patients and SSc-ILD+ patients is shown in table 1. SScILD+ patients were older than SSc-ILD- patients but were not different in terms of gender, height and body mass index. SSc-ILD+ patients more often had diffuse SSc and anti-SCL-70 antibodies than SSc-ILD- patients.

\section{Pulmonary Function Tests}

The main results of the PFTs are shown in table 2. As expected, SSc-ILD+ patients had lower lung volumes compared to SSc-ILD- patients. However, no signs of obstructive airway disease were found in either of the patient groups.

DLCO was impaired in both patient groups as compared with controls. SSc-ILD+ patients had significantly lower DLCO [expressed as percentage of predicted values (\%pred)] than SSc-ILD- patients ( $\mathrm{p}=0.02$; fig. 1$)$. Both SSc-ILD+ and SSc-ILD- patients had reduced DmCO compared to controls. The difference in DmCO between SSc-ILD- patients and controls was modest but significant although lung volumes were similar. DmCO (\%pred) and Vcap (\%pred) were significantly different between the two groups of patients. However, both DmCO and Vcap values overlapped in the two groups of patients, a finding also observable for DLCO and lung volumes (fig. 1). The DmCO:Vcap ratio was much lower in SScILD+ than in SSc-ILD- patients, with minimal overlap (fig. 1). ROC analysis showed that a cutoff value of 0.27 for the DmCO:Vcap ratio identified SSc-ILD+ patients with a sensitivity of $85 \%$ and a specificity of $100 \%$ ( $p<$ 0.0001 ; fig. 2). The sensitivity of DLCO, with a cutoff of $67 \%$ of the predicted value, was $54 \%$, and the overall specificity was $91 \%$ (area under the curve $=0.75, \mathrm{p}=0.005$ ). Comparison of ROC analysis showed that the DmCO: Vcap ratio was more sensitive and specific for identifying SSc-ILD+ than TLC, vital capacity and DLCO ( $<<0.01)$.

\section{Discussion}

The main result of this study is that the DmCO:Vcap ratio was more sensitive and specific than any of the other PFT results for identifying ILD (according to HRCT scan criteria as a diagnostic gold standard) in SSc patients presenting for an annual systematic follow-up.

ILD is present in a large proportion of patients with SSc and constitutes one of the two main causes of death in SSc [1]. ILD is usually slowly progressive in patients with SSc. However, in a small number of cases, it may progress rapidly to end-stage respiratory insufficiency [23]. Rapid detection of the disease is of importance as it should allow for the initiation of treatment (mostly immunosuppressant treatment) with minimal delay [7]. HRCT represents a reliable, noninvasive and reproducible tool for detecting and monitoring ILD in patients with SSc. Since SSc-associated ILD may develop in the 
Fig. 1. TLC, vital capacity (VC), singlebreath lung transfer for CO (TLCO), DmCO, Vcap and the DmCO:Vcap ratio in SSc-ILD- and SSc-ILD+ patients are shown. The bottoms and tops of the boxes represent the lower and upper quartiles, respectively; the bands in the boxes are the median values, and the lower and upper ends of the whiskers are the 10th and 90th percentiles, respectively. Outliers (values that are beyond the 10th or 90th percentile) are represented by circles. Comparisons between groups were made by the Mann-Whitney test. \%theo = Percentage of the theoretical value.

Fig. 2. Comparison of ROC curves corresponding to the sensitivity and specificity of possible cutoff points for vital capacity (VC), single-breath lung transfer for CO (TLCO) and the DmCO:Vcap ratio to discriminate between SSc-ILD- and SScILD+ patients. The DmCO:Vcap ratio was significantly different from vital capacity and single-breath lung transfer for $\mathrm{CO}$ $(\mathrm{p}<0.01) . \%$ theo $=$ Percentage of the theoretical value.
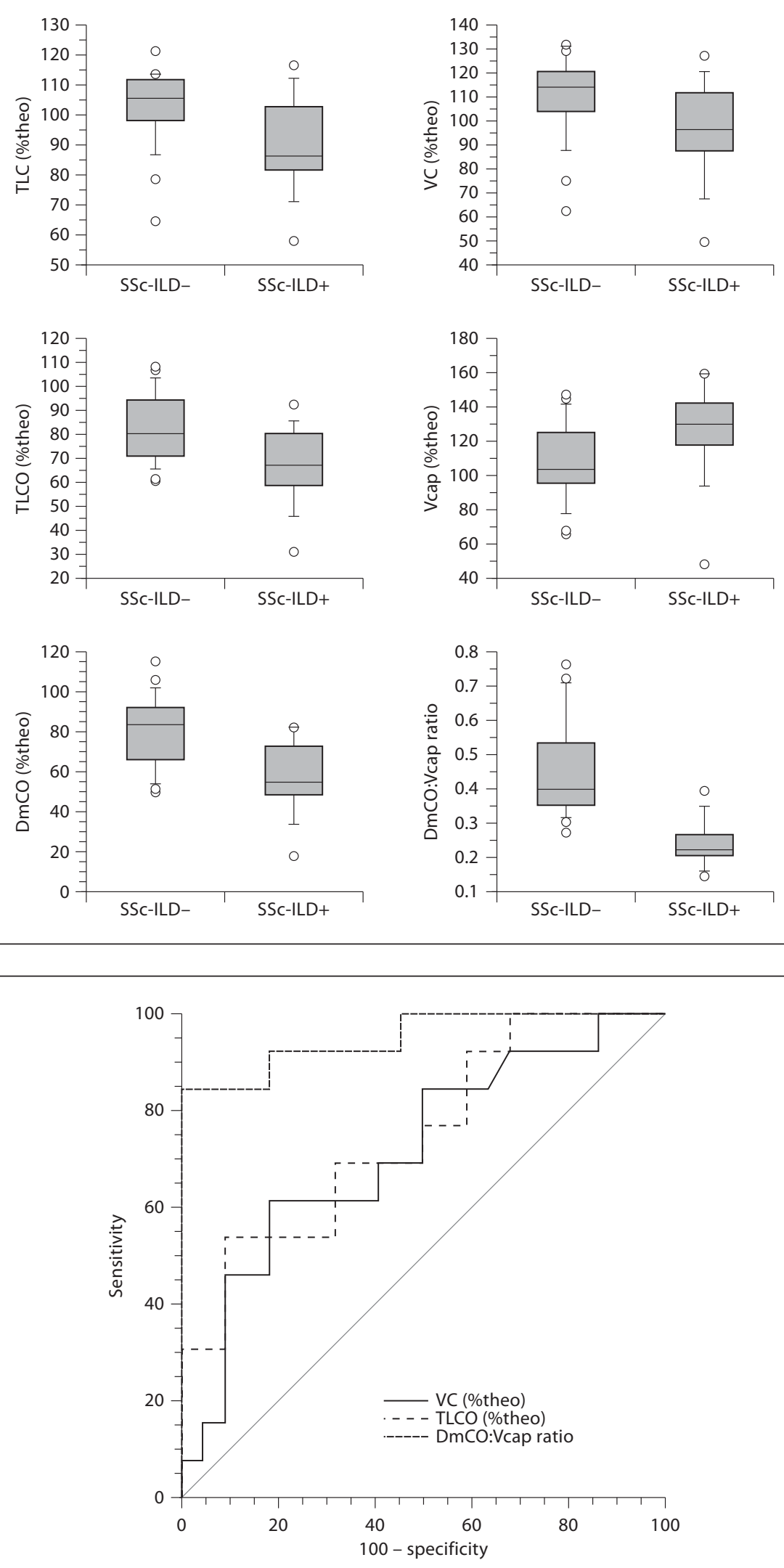
absence of dyspnea, HRCT is recommended in SSc patients, together with PFTs, at the onset of the disease [24]. However, HRCT is not repeated systematically but only when PFT results deteriorate (i.e. values of lung volumes and DLCO) [25]. Nevertheless, some studies have raised concerns about the ability of lung volumes and DLCO to diagnose early forms of ILD in SSc patients, as only approximately $50 \%$ of patients with SSc-ILD diagnosed by HRCT show deteriorated PFT results early in the disease course [8]. Thus, the DmCO:Vcap ratio should represent a routinely available, noninvasive functional test allowing early detection of ILD in SSc patients for early therapeutic purposes.

In our series, we found the classical association between SSc-ILD and diffuse SSc with anti-SCL-70 antibodies. However, some patients with limited SSc and/or with anticentromere antibodies had SSc-ILD. As previously noted, these findings may justify screening for ILD in all SSc patients regardless of the subtype of SSc and antibodies present [25]. In a series of 90 patients with SSc, Launay et al. [25] reported that the majority of them (56\%) presented with HRCT features of ILD. In some series of patients, ILD signs have been identified in up to approximately $90 \%$ of patients with SSc [4]. We found SSc-ILD in only 13 of 35 patients (37\%). It is likely that these differences were due at least in part to the patients' characteristics with regard to the subtypes of SSc. Indeed, ILD is more frequent in diffuse SSc, and this subtype was more frequent in the study by Launay et al. [25] (43\%) than in the current study (34\%). We did not systematically perform a 6-min walk distance test because it has been shown that many confounders and comorbidities, such as pain and musculoskeletal dysfunction, reduce the reliability and validity of this test for SSc [7].

Concerns have been raised previously about the usefulness of the partitioned DLCO for the detection of PAH in SSc patients [14]. Overbeek et al. [14] retrospectively studied 11 SSc patients with PAH, 13 SSc patients without PAH and 10 controls; although DmCO was lower in SSc patients with PAH, the authors found no correlations between DmCO and hemodynamic parameters and therefore concluded that partitioned DLCO was not of interest in the diagnostic workup for PAH in SSc patients. In the study by Overbeek et al. [14], reticular and ground-glass opacities were scored in SSc patients according to the system described by Kazerooni et al. [26]. Fibrosis scores were similar in SSc patients with and without PAH, but the numbers of patients with and without ILD in each group were not given [14]. However, it is likely that the presence of ILD could have interfered with the results of this latter study. In order to avoid confounding factors, we excluded those patients with either PAH and/or heart failure. The rationale for excluding these patients was that DLCO and its components, DmCO and Vcap, are altered by $\mathrm{PAH}$ and $\mathrm{LV}$ failure, rendering it difficult to determine the respective contribution of ILD, PAH and LV failure to the alterations in DLCO, DmCO and Vcap.

We found that DmCO was decreased in the SSc-ILD+ group as compared with both the control and SSc-ILDgroups. This result was expected because a decrease in DmCO is interpreted as a 'thickening' of the alveolocapillary membrane and/or as a decrease in the alveolocapillary membrane surface [16]. In the ILD-SSc+ group, a decrease in membrane surface is likely to be in relationship with the decrease in lung volumes [27]. However, we observed an increase in Vcap in the SSc-ILD+ group compared with the other two groups. This finding was unexpected, as a decrease in the alveolo-capillary membrane surface has been shown in some conditions to lead to a decreased blood volume [27]. One can speculate that this observation is due to the fact that the SSc-ILD- group had more patients with limited SSc with preclinical vasculopathy and thus reduced Vcap. This hypothesis is unlikely because Vcap (\%pred) was not significantly different between patients with limited and diffuse SSc (data not shown). Theoretically, an association between a low DLCO, a low DmCO and a high Vcap can be found in the case of high pulmonary venous pressure, such as in LV failure, pulmonary veno-occlusive disease and/or compression of the pulmonary veins [28]. In our patients, pulmonary vein compression was excluded by means of HRCT. However, because of the high prevalence of diastolic left heart dysfunction and pulmonary veno-occlusive disease in SSc, it is not possible to definitively rule out the hypothesis that a mild form of diastolic left heart dysfunction or pulmonary veno-occlusive disease, unable to alter Doppler echocardiography and/or RHC, may have been involved in our patients. As an example, in the algorithm published by Hachulla et al. [10], RHC is not performed in patients with a tricuspid regurgitant velocity $<2.5 \mathrm{~m} / \mathrm{s}$. Thus, this algorithm is unable to indicate whether or not some SSc patients with a tricuspid regurgitant velocity $<2.5$ $\mathrm{m} / \mathrm{s}$ have pulmonary hypertension.

As discussed elsewhere, the acquisition of DmCO might be prone to inaccuracy, as a small change in the slope of the $1 /$ DLCO-1/ $\theta$ line can lead to a large change at the y-intercept that determines DmCO [14]. However, this potential error is likely to be systematic and thus unlikely to significantly bias the different value spans in the 
patient groups. In addition, duplicate measurements were performed in order to reduce the uncertainty of measures. An alternate method consisting of simultaneously measuring DLCO with the lung diffusing capacity for nitric oxide has been developed in order to overcome some of the inaccuracy in the acquisition of DmCO [29]. Calculation of DmCO and Vcap with this latter method has been recently discussed and remains debated [30, 31]. Nevertheless, practical measuring equipment that gives quick and reproducible results for simultaneous singlebreath DLCO and lung diffusing capacity for nitric oxide is being developed, and the clinical applications of this technique should be studied in patients with SSc.
Although the results reported here are promising, they need to be validated by prospectively studying a new cohort. However, as SSc is not a frequent disease, such a validation in our single center is not feasible and requires a multicenter prospective study using the criteria defined from the data we have shown.

In conclusion, we found that the DmCO:Vcap ratio was more sensitive and specific than any of the other PFT results for identifying patients with HRCT signs of SScILD among SSc patients without PAH and/or LV failure. The clinical usefulness of a screening procedure including the DmCO:Vcap ratio in SSc patients remains to be established by a prospective validation study.

\section{References}

1 Steen VD, Medsger TA: Changes in causes of death in systemic sclerosis, 1972-2002. Ann Rheum Dis 2007;66:940-944.

2 Damjanov N, Ostojic P, Kaloudi O, Alari S, Guiducci S, Stanflin N, Nestorovic B, Knezevic J, Camiciottoli G, Porta F, Pistolesi M, Ibba-Manneschi L, Conforti ML, Candelieri A, Matucci Cerinic M: Induced sputum in systemic sclerosis interstitial lung disease: comparison to healthy controls and bronchoalveolar lavage. Respiration 2009;78:5662.

3 Wells AU, Rubens MB, du Bois RM, Hansell DM: Serial CT in fibrosing alveolitis: prognostic significance of the initial pattern. AJR Am J Roentgenol 1993;161:1159-1165.

-4 Schurawitzki H, Stiglbauer R, Graninger W, Herold C, Polzleitner D, Burghuber OC, Tscholakoff D: Interstitial lung disease in progressive systemic sclerosis: high-resolution CT versus radiography. Radiology 1990; 176:755-759.

5 Diot E, Boissinot E, Asquier E, Guilmot JL, Lemarie E, Valat C, Diot P: Relationship between abnormalities on high-resolution CT and pulmonary function in systemic sclerosis. Chest 1998; 114:1623-1629.

6 de Souza RB, Borges CT, Capelozzi VL, Parra ER, Jatene FB, Kavakama J, Kairalla RA, Bonfa E: Centrilobular fibrosis: an underrecognized pattern in systemic sclerosis. Respiration 2009;77:389-397.

7 Bussone G, Mouthon L: Interstitial lung disease in systemic sclerosis. Autoimmun Rev 2011;10:248-255.

$\checkmark 8$ Wells AU, Cullinan P, Hansell DM, Rubens MB, Black CM, Newman-Taylor AJ, Du Bois RM: Fibrosing alveolitis associated with systemic sclerosis has a better prognosis than lone cryptogenic fibrosing alveolitis. Am J Respir Crit Care Med 1994;149:1583-1590.
9 Wells AU: High-resolution computed tomography and scleroderma lung disease. Rheumatology (Oxford) 2008; 47(suppl 5):v59-v61.

10 Hachulla E, Gressin V, Guillevin L, Carpentier P, Diot E, Sibilia J, Kahan A, Cabane J, Frances C, Launay D, Mouthon L, Allanore Y, Tiev KP, Clerson P, de Groote P, Humbert M: Early detection of pulmonary arterial hypertension in systemic sclerosis: a French nationwide prospective multicenter study. Arthritis Rheum 2005;52:3792-3800.

11 de Groote P, Gressin V, Hachulla E, Carpentier P, Guillevin L, Kahan A, Cabane J, Frances C, Lamblin N, Diot E, Patat F, Sibilia J, Petit H, Cracowski JL, Clerson P, Humbert M: Evaluation of cardiac abnormalities by doppler echocardiography in a large nationwide multicentric cohort of patients with systemic sclerosis. Ann Rheum Dis 2008;67: 31-36.

$\checkmark 12$ Agostoni P, Bussotti M, Cattadori G, Margutti E, Contini M, Muratori M, Marenzi G, Fiorentini C: Gas diffusion and alveolar-capillary unit in chronic heart failure. Eur Heart J 2006;27:2538-2543.

13 Steen V, Medsger TA Jr: Predictors of isolated pulmonary hypertension in patients with systemic sclerosis and limited cutaneous involvement. Arthritis Rheum 2003;48:516522.

14 Overbeek MJ, Groepenhoff H, Voskuyl AE, Smit EF, Peeters JW, Vonk-Noordegraaf A, Spreeuwenberg MD, Dijkmans BC, Boonstra A: Membrane diffusion- and capillary blood volume measurements are not useful as screening tools for pulmonary arterial hypertension in systemic sclerosis: a case control study. Respir Res 2008;9:68.
15 Roughton FJ, Forster RE: Relative importance of diffusion and chemical reaction rates in determining rate of exchange of gases in the human lung, with special reference to true diffusing capacity of pulmonary membrane and volume of blood in the lung capillaries. J Appl Physiol 1957;11:290-302.

16 Degano B, Mittaine M, Guenard H, Rami J, Garcia G, Kamar N, Bureau C, Peron JM, Rostaing L, Riviere D: Nitric oxide and carbon monoxide lung transfer in patients with advanced liver cirrhosis. J Appl Physiol 2009; 107:139-143.

17 LeRoy EC, Medsger TA Jr: Criteria for the classification of early systemic sclerosis. J Rheumatol 2001;28:1573-1576.

-18 Zanen P, van der Lee L, van der Mark T, van den Bosch JM: Reference values for alveolar membrane diffusion capacity and pulmonary capillary blood volume. Eur Respir J 2001;18:764-769.

19 Goh NS, Desai SR, Veeraraghavan S, Hansell DM, Copley SJ, Maher TM, Corte TJ, Sander CR, Ratoff J, Devaraj A, Bozovic G, Denton CP, Black CM, du Bois RM, Wells AU: Interstitial lung disease in systemic sclerosis: a simple staging system. Am J Respir Crit Care Med 2008;177:1248-1254.

20 Bonay M, Bancal C, de Zuttere D, Arnoult F, Saumon G, Camus F: Normal pulmonary capillary blood volume in patients with chronic infiltrative lung disease and high pulmonary artery pressure. Chest 2004;126: 1460-1466.

21 van der Lee I, Zanen P, Grutters JC, Snijder RJ, van den Bosch JM: Diffusing capacity for nitric oxide and carbon monoxide in patients with diffuse parenchymal lung disease and pulmonary arterial hypertension. Chest 2006;129:378-383. 
22 Machin D, Campbell M, Fayers P, Pinol A: Sample Size Tables for Clinical Studies. Malden, Blackwell Science, 1997.

-23 Steen VD, Conte C, Owens GR, Medsger TA Jr: Severe restrictive lung disease in systemic sclerosis. Arthritis Rheum 1994;37:12831289.

24 Mouthon L, Berezne A, Guillevin L, Valeyre D: Therapeutic options for systemic sclerosis related interstitial lung diseases. Respir Med 2010;104(suppl 1):S59-S69.

25 Launay D, Remy-Jardin M, Michon-Pasturel U, Mastora I, Hachulla E, Lambert M, Delannoy V, Queyrel V, Duhamel A, Matran R, De Groote P, Hatron PY: High resolution computed tomography in fibrosing alveolitis associated with systemic sclerosis. J Rheumatol 2006;33:1789-1801.
6 Kazerooni EA, Martinez FJ, Flint A, Jamadar DA, Gross BH, Spizarny DL, White RI, Lynch JP, Toews G: Thin-section CT obtained at $10-\mathrm{mm}$ increments versus limited three-level thin-section CT for idiopathic pulmonary fibrosis: correlation with pathologic scoring. AJR Am J Roentgenol 1997; 169:977-983.

27 Glenet SN, De Bisschop C, Vargas F, Guenard HJ: Deciphering the nitric oxide to carbon monoxide lung transfer ratio: physiological implications. J Physiol 2007;582:767775.

28 Guazzi M, Pontone G, Brambilla R, Agostoni P, Reina G: Alveolar-capillary membrane gas conductance: a novel prognostic indicator in chronic heart failure. Eur Heart J 2002;23: 467-476.
29 Guenard H, Varene N, Vaida P: Determination of lung capillary blood volume and membrane diffusing capacity in man by the measurements of $\mathrm{NO}$ and $\mathrm{CO}$ transfer. Respir Physiol 1987;70:113-120.

30 Borland CD, Dunningham H, Bottrill F, Vuylsteke A, Yilmaz C, Dane DM, Hsia CC: Significant blood resistance to nitric oxide transfer in the lung. J Appl Physiol 2010;108: 1052-1060.

31 Ceridon ML, Beck KC, Olson TP, Bilezikian JA, Johnson BD: Calculating alveolar capillary conductance and pulmonary capillary blood volume: comparing the multiple- and single-inspired oxygen tension methods. J Appl Physiol 2010;109:643-653. 